\title{
MICROLENSING DISCOVERY OF A TIGHT, LOW-MASS-RATIO PLANETARY-MASS OBJECT AROUND AN OLD FIELD BROWN DWARF
}

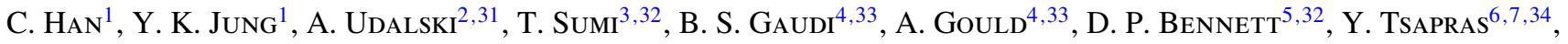 \\ AND
}

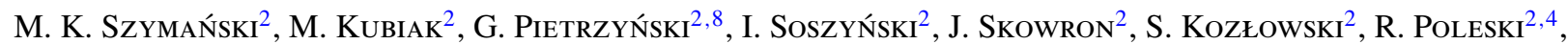
K. UlACZYK ${ }^{2}$, Ł. WyrzYKOWSKI ${ }^{2,9}$, P. PIETRUKOWICZ ${ }^{2}$

(The OGLE COLlaboration)

F. Abe ${ }^{10}$, I. A. Bond ${ }^{11}$, C. S. Botzler ${ }^{12}$, P. Chote ${ }^{13}$, M. Freeman $^{12}$, A. Fukui ${ }^{14}$, K. Furusawa ${ }^{10}$, P. Harris ${ }^{13}$, Y. Itow $^{10}$, C. H. Ling $^{11}$, K. Masuda ${ }^{10}$, Y. Matsubara ${ }^{10}$, Y. Muraki ${ }^{10}$, K. Ohnishi ${ }^{15}$, N. J. Rattenbury ${ }^{12}$, To. Saito ${ }^{16}$, D. J. Sullivan ${ }^{13}$, W. L. Sweatman ${ }^{11}$, D. Suzuki ${ }^{3}$, P. J. Tristram ${ }^{17}$, K. Wada ${ }^{3}$, P. C. M. Yock ${ }^{12}$

(The MOA COLlaboration)

V. Batista ${ }^{4}$, G. Christie ${ }^{18}$, J.-Y. Choi ${ }^{1}$, D. L. DePoy ${ }^{19}$, Subo Dong ${ }^{20}$, K.-H. Hwang ${ }^{1}$, A. Kavka ${ }^{4}$, C.-U. LeE ${ }^{21}$,

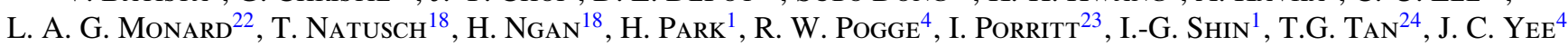
(THE $\mu$ FUN COLLABORATION)

K. A. Alsubai ${ }^{25}$, V. Bozza ${ }^{26}$, D. M. Bramich ${ }^{27}$, P. Browne ${ }^{28}$, M. Dominik ${ }^{28}$, K. Horne $^{28}$, M. Hundertmark ${ }^{28}$,

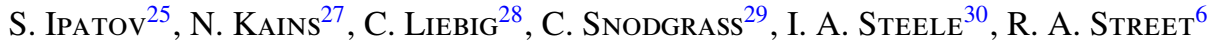

(The Robonet Collaboration)

${ }^{1}$ Department of Physics, Chungbuk National University, Cheongju 371-763, Korea

${ }^{2}$ Warsaw University Observatory, Al. Ujazdowskie 4, 00-478 Warszawa, Poland

${ }^{3}$ Department of Earth and Space Science, Osaka University, Osaka 560-0043, Japan

${ }^{4}$ Department of Astronomy, Ohio State University, 140 West 18th Avenue, Columbus, OH 43210, USA

${ }^{5}$ University of Notre Dame, Department of Physics, 225 Nieuwland Science Hall, Notre Dame, IN 46556-5670, USA

${ }^{6}$ Las Cumbres Observatory Global Telescope Network, 6740B Cortona Dr, Goleta, CA 93117, USA

${ }^{7}$ School of Physics and Astronomy, Queen Mary University of London, Mile End Road, London E1 4NS, UK

${ }^{8}$ Universidad de Concepción, Departamento de Astronomia, Casilla 160-C, Concepción, Chile

${ }^{9}$ Institute of Astronomy, University of Cambridge, Madingley Road, Cambridge CB3 OHA, UK

${ }^{10}$ Solar-Terrestrial Environment Laboratory, Nagoya University, Nagoya 464-8601, Japan

${ }^{11}$ Institute of Information and Mathematical Sciences, Massey University, Private Bag 102-904, North Shore Mail Centre, Auckland, New Zealand

${ }^{12}$ Department of Physics, University of Auckland, Private Bag 92-019, Auckland 1001, New Zealand

${ }^{13}$ School of Chemical and Physical Sciences, Victoria University, Wellington, New Zealand

${ }^{14}$ Okayama Astrophysical Observatory, National Astronomical Observatory of Japan, Asakuchi, Okayama 719-0232, Japan

${ }_{15}^{15}$ Nagano National College of Technology, Nagano 381-8550, Japan

${ }^{16}$ Tokyo Metropolitan College of Aeronautics, Tokyo 116-8523, Japan

${ }^{17}$ Mt. John University Observatory, P.O. Box 56, Lake Tekapo 8770, New Zealand

${ }^{18}$ Auckland Observatory, Auckland, New Zealand

${ }^{19}$ Department of Physics and Astronomy, Texas A\&M University, College Station, TX 77843, USA

${ }^{20}$ Institute for Advanced Study, Einstein Drive, Princeton, NJ 08540, USA

${ }^{21}$ Korea Astronomy and Space Science Institute, Daejeon 305-348, Korea

${ }^{22}$ Klein Karoo Observatory, Calitzdorp, and Bronberg Observatory, Pretoria, South Africa

${ }^{23}$ Turitea Observatory, Palmerston North, New Zealand

${ }^{24}$ Perth Exoplanet Survey Telescope, Perth, Australia

${ }^{25}$ Alsubai Establishment for Scientific Studies, Doha, Qatar

${ }^{26}$ Università degli Studi di Salerno, Dipartimento di Fisica "E.R. Caianiello," Via S. Allende, I-84081 Baronissi (SA), Italy

${ }^{27}$ European Southern Observatory, Karl-Schwarzschild-Str. 2, D-85748 Garching bei München, Germany

${ }^{28}$ SUPA, School of Physics \& Astronomy, University of St Andrews, North Haugh, St. Andrews KY16 9SS, UK

${ }^{29}$ Max Planck Institute for Solar System Research, Max-Planck-Str. 2, D-37191 Katlenburg-Lindau, Germany

${ }^{30}$ Astrophysics Research Institute, Liverpool John Moores University, Liverpool CH41 1LD, UK Received 2013 July 24; accepted 2013 September 18; published 2013 October 31

\section{ABSTRACT}

Observations of accretion disks around young brown dwarfs (BDs) have led to the speculation that they may form planetary systems similar to normal stars. While there have been several detections of planetary-mass objects around BDs (2MASS 1207-3932 and 2MASS 0441-2301), these companions have relatively large mass ratios and projected separations, suggesting that they formed in a manner analogous to stellar binaries. We present the discovery of a planetary-mass object orbiting a field BD via gravitational microlensing, OGLE-2012-BLG-0358Lb. The system is a low secondary/primary mass ratio $(0.080 \pm 0.001)$, relatively tightly separated $(\sim 0.87 \mathrm{AU})$ binary composed of a planetary-mass object with $1.9 \pm 0.2$ Jupiter masses orbiting a BD with a mass $0.022 M_{\odot}$. The relatively small mass ratio and separation suggest that the companion may have formed in a protoplanetary disk around the $\mathrm{BD}$ host in a manner analogous to planets.

Key words: brown dwarfs - gravitational lensing: micro - planetary systems

Online-only material: color figures 


\section{INTRODUCTION}

Brown dwarfs (BDs) are sub-stellar objects that are too low in mass to sustain hydrogen fusion reactions in their cores. Although still a matter of debate, the most popular theory about the origin of BDs is that they form via direct collapse, similar to stars, and perhaps aided by turbulent fragmentation (see Luhman 2012 for a review). This theory is supported by observational evidence showing that several medium-sized BDs are girdled by disks of material (Luhman et al. 2005; Apai et al. 2005; Ricci et al. 2012). The existence of accretion disks around these failed stars naturally leads to the speculation that BDs may also harbor planetary systems analogous to those found in abundance around stars.

There have been several detections of planetary-mass objects around BDs: 2MASS 1207-3932B with $M_{\mathrm{B}} \sim 4 M_{\mathrm{J}}$ (Chauvin et al. 2004) and 2MASS 0441-2301B with $M_{\mathrm{B}} \sim 7.5 M_{\mathrm{J}}$ (Todorov et al. 2010). However, these systems have relatively large mass ratios of $q \sim 0.16$ for 2MASS 1207-3932 and $q \sim 0.25-0.5$ for 2MASS 0441-2301, more akin to binary stellar systems. Furthermore, they have relatively large separations ( $\sim 15$ AU for 2MASS $0441-2301$ and $\sim 45$ AU for 2MASS 12073932), likely near or beyond the outer edges of the accretion disks observed around BDs (Luhman et al. 2007; Ricci et al. 2012, 2013). Therefore, it seems unlikely that these companions formed from the protoplanetary disk material via either of the popular giant planet formation mechanisms of core accretion (Pollack et al. 1996) or disk fragmentation (Kuiper 1951; Cameron 1978; Boss 1997; Durisen et al. 2007). Rather, these are more likely to have formed in the same way as stellar binaries through the process of gravitational fragmentation of massive primordial disks (Lodato et al. 2005). Thus, according to a classification system based on their formation, they are not bona fide planets.

In this paper, we report a microlensing discovery of a tight, low-mass-ratio planetary-mass object orbiting an old field BD that we suggest may have formed in a protoplanetary disk. Microlensing is the astronomical phenomenon wherein the brightness of a star is magnified by the bending of light due to the gravity of an intervening object (lens) positioned between the background star (source) and an observer. This discovery was possible, despite the extremely low luminosity of the BD, because the lensing phenomenon occurs regardless of the lens brightness.

\section{OBSERVATION}

The BD planetary system was discovered in the microlensing event OGLE-2012-BLG-0358. The event occurred in the 2012 observing season on a star located in the Galactic bulge field with equatorial coordinates (R.A., decl.) $)_{2000}=$ $\left(17^{\mathrm{h}} 42^{\mathrm{m}} 46^{\mathrm{s}} .77,-24^{\circ} 15^{\prime} 39^{\prime} \cdot 6\right)$, which corresponds to the Galactic coordinates $(l, b)_{2000}=(3.65,2.99)$. It was first discovered by the Optical Gravitational Lensing Experiment (OGLE; Udalski 2003) group in 2012 April. During its early phase, the light curve of the event appeared to be a high-magnification event produced by a single mass. Since high-magnification events are prime targets for planet detections, the event was additionally observed by other groups, including the Microlensing

\footnotetext{
31 The OGLE Collaboration.

32 The MOA Collaboration.

33 The $\mu$ FUN Collaboration.

34 The RoboNet Collaboration.
}

Table 1

Telescopes

\begin{tabular}{ll}
\hline \hline Group & \multicolumn{1}{c}{ Telescope } \\
\hline OGLE & $1.3 \mathrm{~m}$ Warsaw, Las Campanas, Chile \\
MOA & $0.6 \mathrm{~m}$ Boller \& Chivens, Mt. John, New Zealand \\
$\mu$ FUN & $1.3 \mathrm{~m}$ SMARTS, Cerro Tololo Inter-American (CTIO), Chile \\
$\mu$ FUN & $0.4 \mathrm{~m}$ Auckland, New Zealand \\
$\mu$ FUN & 0.36 m Klein Karoo Observatory (KKO), South Africa \\
$\mu$ FUN & 0.3 m Perth Extrasolar Survey Telescope (PEST), Australia \\
$\mu$ FUN & $0.4 \mathrm{~m}$ Turitea, New Zealand \\
RoboNet & $2.0 \mathrm{~m}$ Faulkes North Telescope (FTN), Hawaii, USA \\
RoboNet & $2.0 \mathrm{~m}$ Faulkes South Telescope (FTS), Australia \\
RoboNet & $2.0 \mathrm{~m}$ Liverpool Telescope (LT), Canary Islands, Spain \\
\hline
\end{tabular}

Follow-Up Network ( $\mu$ FUN; Gould et al. 2006), Microlensing Observations in Astrophysics (MOA; Bond et al. 2001; Sumi et al. 2003), and RoboNet (Tsapras et al. 2009). As the event approached its peak, it was noted that the light curve deviated from a standard single-lens light curve and the anomaly became obvious as the light curve peaked again five days after the first peak. Continued observations by the OGLE group revealed that the event produced another extended weak bump. In Table 1, we list the telescopes used for observation.

In Figure 1, we present the light curve of the event. It is characterized by two strong peaks centered at Heliocentric Julian Date (HJD) 2,456,537.5 and 2,456,542.5 and an extended weak bump centered at HJD $\sim 2,456,065$. A strong peak in a lensing light curve occurs when a source star approaches close to or crosses the tip of a caustic produced by a lens composed of multiple objects. The caustic represents the envelope of light rays refracted by a curved surface and it is commonly visible as a curved region of bright light appearing when light shines on a drinking glass. For a gravitational lens composed of two masses, caustics form a single or multiple sets of closed curves, each of which is composed of concave curves that meet at cusps.

\section{MODELING}

With the signature of lens multiplicity, we conduct binarylens modeling of the observed light curve. A basic description of a binary-lens light curve requires seven lensing parameters. Three of these parameters describe the lens-source approach, including the time of the closest source approach to a reference position of the binary lens, $t_{0}$, the separation between the source and the reference position, $u_{0}$ (normalized by the angular Einstein radius $\theta_{\mathrm{E}}$ ), and the timescale for the source to cross $\theta_{\mathrm{E}}$ (Einstein timescale $t_{\mathrm{E}}$ ). The Einstein ring denotes the image of a source in the event of perfect lens-source alignment, and its radius is commonly used as the length scale of lensing phenomena. Another three lensing parameters describe the binary nature of the lens, including the projected separation, $s$ (normalized by $\theta_{\mathrm{E}}$ ), the mass ratio, $q$, between the binary components, and the angle between the source trajectory and the binary axis, $\alpha$ (source-trajectory angle). The last parameter is the normalized source radius $\rho_{*}=\theta_{*} / \theta_{\mathrm{E}}$, where $\theta_{*}$ is the angular source radius. This parameter is needed to describe precisely the parts of a lensing light curve involved with caustic crossings or approaches of the source during which the lensing light curve is affected by the finite size of the source star. In our modeling of finite-source effects, we additionally consider the limb-darkening variation of the source star 


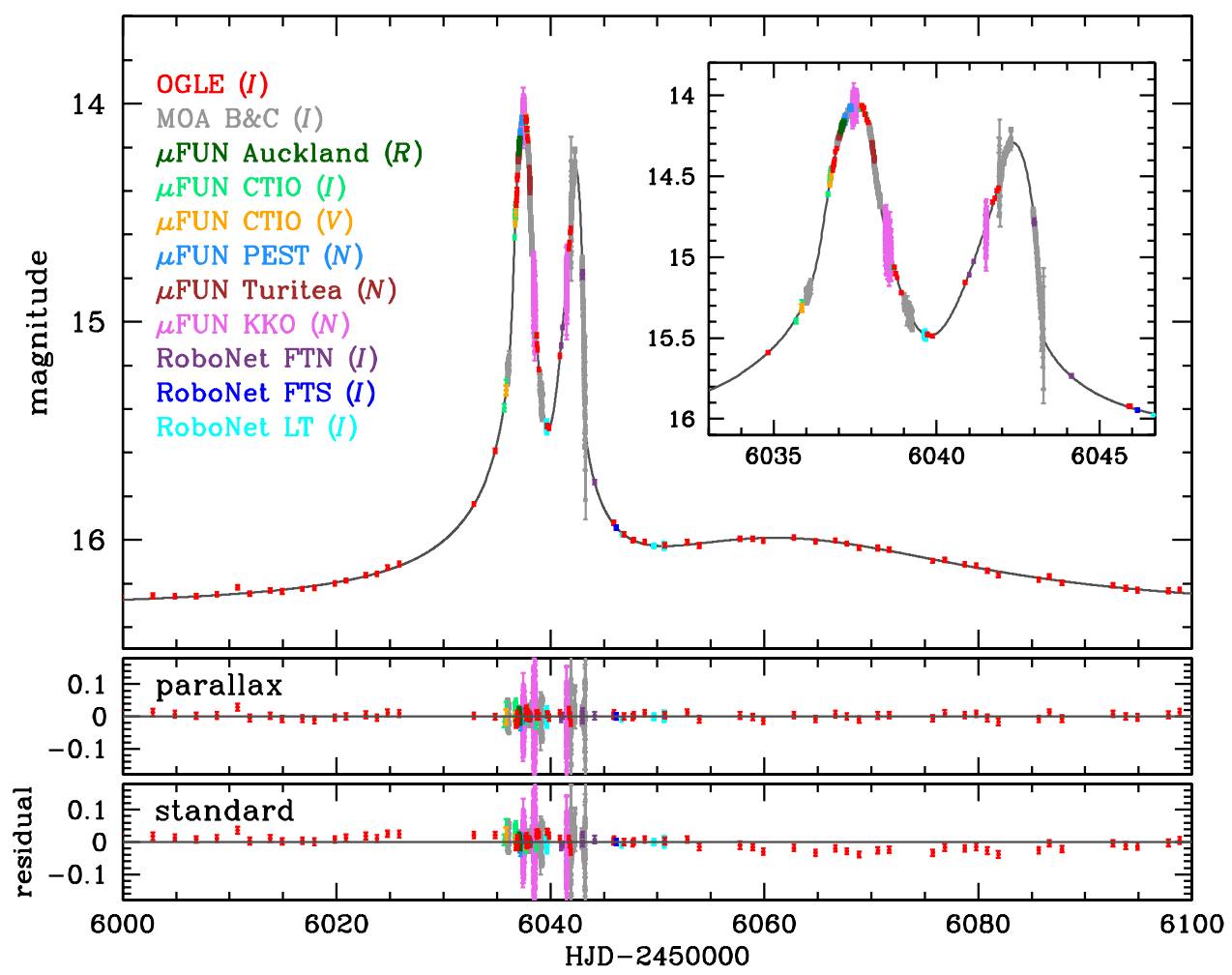

Figure 1. Light curve of the microlensing event OGLE-2012-BLG-0358. The lower two panels show the residuals from the best-fit standard binary-lens model and from the model considering the parallax effect. The letters after the individual telescopes represent the pass bands of observation.

(A color version of this figure is available in the online journal.)

surface by modeling the surface brightness profile as a standard linear law.

We search for a solution of lensing parameters that best describes the observed light curve by minimizing $\chi^{2}$ in the parameter space encompassing wide ranges of binary separations and mass ratios. For $\chi^{2}$ minimization, we use the Markov Chain Monte Carlo method. In order to properly combine data sets obtained from different observatories, we readjust photometric errors of the individual data sets first by adding a quadratic error term so that the cumulative distribution of $\chi^{2}$ ordered by magnifications matches a standard cumulative distribution of Gaussian errors and then by rescaling errors so that $\chi^{2}$ per degree of freedom becomes unity for each data set. We eliminate data points with large errors and obvious outliers to minimize their effect on modeling.

From the initial search for solutions obtained from modeling based on the standard binary-lensing parameters (standard model), we find a solution of a binary lens with a projected separation $s \sim 1.7$ and a mass ratio $q \sim 9$.8. See Table 2 for the complete solution. Although the model describes the main feature of the two strong peaks, it is found that there exist longterm residuals in the wings of the light curve, including the extended weak bump as shown in the bottom panel of Figure 1. This suggests the need to consider higher-order effects.

Several causes of long-term deviations in lensing light curves exist. The first cause is the change of the observer's position caused by the orbital motion of the Earth around the Sun (Gould 1992; Alcock et al. 1995). This "parallax effect" causes the source trajectory to deviate from rectilinear, resulting in longterm deviations. The second is the positional change of the lens caused by the orbital motion of the binary lens (Dominik 1998; Albrow et al. 2000; Bennett et al. 2010; Penny et al. 2011; Shin et al. 2011, 2012; Skowron et al. 2011). In addition to causing the source trajectory to deviate from rectilinear, the "lens orbital effect" causes further deviation in lensing light curves by deforming the caustic over the course of the event. The last cause of the deviation is the change of the source position caused by its orbital motion, if the source is a binary (Han \& Gould 1997; Dominik 1998). Since this affects lensing light curves in a way similar to the parallax effect, it is often referred to as the "xallarap effect," which is parallax spelled backward.

Considering the parallax effect requires two parameters, $\pi_{\mathrm{E}, N}$ and $\pi_{\mathrm{E}, E}$, which represent the two components of the lens parallax vector $\boldsymbol{\pi}_{\mathrm{E}}$ projected onto the sky along the north and east equatorial coordinates, respectively. The magnitude of the parallax vector corresponds to the relative lens-source parallax, $\pi_{\text {rel }}=\mathrm{AU}\left(D_{\mathrm{L}}^{-1}-D_{\mathrm{S}}^{-1}\right)$, scaled to the Einstein radius of the lens, i.e., $\pi_{\mathrm{E}}=\pi_{\mathrm{rel}} / \theta_{\mathrm{E}}$ (Gould 2004). To first order approximation, the lens orbital motion is described by two parameters, $d s / d t$ and $d \alpha / d t$, which represent the change rates of the normalized binary separation and the source-trajectory angle, respectively (Albrow et al. 2000). Modeling the xallarap effect requires five parameters: the components of the xallarap vector, $\xi_{\mathrm{E}, N}$ and $\xi_{\mathrm{E}, N}$, the orbital period, $P$, inclination, $i$, and the phase angle, $\psi$, of the source orbital motion. The magnitude of the xallarap vector, $\xi_{\mathrm{E}}$, corresponds to the semi-major axis of the source's orbital motion with respect to the center of mass normalized by the projected Einstein radius onto the source plane (Dong et al. 2009).

\section{RESULTS}

We test models considering the higher-order effects and the results are summarized in Table 2. By comparing the results, our findings are as follows. First, it is found that the parallax effect substantially improves the fit as shown by the residuals 
Table 2

Lensing Parameters

\begin{tabular}{|c|c|c|c|c|c|}
\hline \multirow[t]{2}{*}{ Parameters } & \multicolumn{5}{|c|}{ Model } \\
\hline & Standard & Parallax $\left(u_{0}>0\right)$ & Parallax $\left(u_{0}<0\right)$ & Orbit + Parallax & Xallarap $(P=1 \mathrm{yr})$ \\
\hline$\chi^{2} /$ dof & $2347.81 / 1592$ & $1598.22 / 1590$ & $1596.24 / 1590$ & $1595.17 / 1588$ & $1601.53 / 1588$ \\
\hline$t_{0}\left(\mathrm{HJD}^{\prime}\right)$ & $6040.24 \pm 0.01$ & $6040.33 \pm 0.01$ & $6040.33 \pm 0.01$ & $6057.49 \pm 0.10$ & $6040.33 \pm 0.01$ \\
\hline$u_{0}$ & $0.108 \pm 0.001$ & $0.098 \pm 0.001$ & $-0.098 \pm 0.001$ & $-0.832 \pm 0.002$ & $0.098 \pm 0.001$ \\
\hline$t_{\mathrm{E}}$ (days) & $24.38 \pm 0.07$ & $26.47 \pm 0.11$ & $26.46 \pm 0.11$ & $25.64 \pm 0.08$ & $26.46 \pm 0.12$ \\
\hline$s_{1}$ & $1.687 \pm 0.002$ & $1.696 \pm 0.003$ & $1.696 \pm 0.003$ & $1.700 \pm 0.002$ & $1.696 \pm 0.003$ \\
\hline$q_{1}\left(10^{-2}\right)$ & $9.810 \pm 0.071$ & $12.531 \pm 0.154$ & $12.486 \pm 0.159$ & $12.281 \pm 0.118$ & $12.487 \pm 0.179$ \\
\hline$\alpha$ & $5.544 \pm 0.001$ & $-0.721 \pm 0.001$ & $0.722 \pm 0.001$ & $-5.427 \pm 0.002$ & $5.562 \pm 0.001$ \\
\hline$\rho_{\star}\left(10^{-3}\right)$ & $2.64 \pm 0.01$ & $2.36 \pm 0.01$ & $2.37 \pm 0.01$ & $2.38 \pm 0.01$ & $2.36 \pm 0.01$ \\
\hline$\pi_{\mathrm{E}, N}$ & $\ldots$ & $-1.42 \pm 0.06$ & $1.49 \pm 0.07$ & $1.45 \pm 0.03$ & $\ldots$ \\
\hline$\pi_{\mathrm{E}, E}$ & $\cdots$ & $-0.34 \pm 0.04$ & $-0.19 \pm 0.06$ & $-0.38 \pm 0.02$ & $\cdots$ \\
\hline$d s / d t\left(\mathrm{yr}^{-1}\right)$ & $\cdots$ & $\ldots$ & $\ldots$ & $0.05 \pm 0.01$ & $\cdots$ \\
\hline$d \alpha / d t\left(\mathrm{yr}^{-1}\right)$ & $\cdots$ & $\cdots$ & $\cdots$ & $-0.04 \pm 0.01$ & $\ldots$ \\
\hline$\xi_{\mathrm{E}, N}$ & $\cdots$ & $\cdots$ & $\cdots$ & $\ldots$ & $-2.18 \pm 0.03$ \\
\hline$\xi_{\mathrm{E}, E}$ & $\cdots$ & $\cdots$ & $\cdots$ & $\cdots$ & $0.19 \pm 0.11$ \\
\hline$\psi(\operatorname{deg})$ & $\ldots$ & $\ldots$ & $\ldots$ & $\ldots$ & $247.8 \pm 2.0$ \\
\hline$\ell(\operatorname{deg})$ & $\cdots$ & $\cdots$ & $\cdots$ & $\cdots$ & $10.3 \pm 1.9$ \\
\hline
\end{tabular}

Notes. HJD' $=$ HJD-2,450,000. We note that the lensing parameters $t_{0}$ and $u_{0}$ are measured with respect to the center of the caustic located on the planet side.

in Figure 1. We find that the improvement is $\Delta \chi^{2} \sim 752$ compared to the standard binary-lens model. Second, when we additionally consider the lens orbital effect, on the other hand, the improvement of the fit $\Delta \chi^{2} \sim 1$ is meager. Finally, we find that considering the xallarap effect yields solutions as good as the parallax solution for source orbital periods $P>0.6$ yr. This is expected because it is known that xallarap effects can mimic parallax effects (Smith et al. 2003; Dong et al. 2009). However, the xallarap solutions result in masses of the source companion bigger than $3 M_{\odot}$. This contradicts the upper limit set by the observed blended light unless the source companion is a black hole and thus we exclude the xallarap interpretation. Therefore, we conclude that the dominant effect for the long-term deviation is the parallax effect. Finally, since the source lies very near the ecliptic, it is subject to the "ecliptic degeneracy," which has almost identical parameters except $\left(u_{0}, \alpha, \pi_{\mathrm{E}, N}\right) \rightarrow-\left(u_{0}, \alpha, \pi_{\mathrm{E}, N}\right)$ (Skowron et al. 2011).

In Figure 1, we present the best-fit model (parallax model in Table 2) curve which is overplotted on the observed light curve. In Figure 2, we also present the geometry of the lens system for the best-fit solution. It is found that the lens consists of binary components with a projected separation bigger than the Einstein radius corresponding to the total mass of the binary. For such a binary lens, there exist two sets of four-cusp caustics, where one small set is located close to the heavier lens component (primary) and the other bigger set is located toward the lowermass lens component (companion). The event was produced by the source trajectory passing the tips of the caustic located on the companion side. The strong peaks at HJD 2,456,537.5 and 2,456,542.5 were produced at the moments of the source crossings over the caustic tips, while the extended weak bump centered at HJD 2,456,065 was produced as the source passed through the magnification zone of the primary lens. Despite the relatively short time scale $t_{\mathrm{E}} \sim 26.5$ days of the event, a clear detection of the parallax effect was possible due to a combination of the large value of the lens parallax and the good coverage of the extended bump that continued for almost two months after the main peaks.

Detecting the parallax effect is important for the determinations of the physical lens parameters because the lens

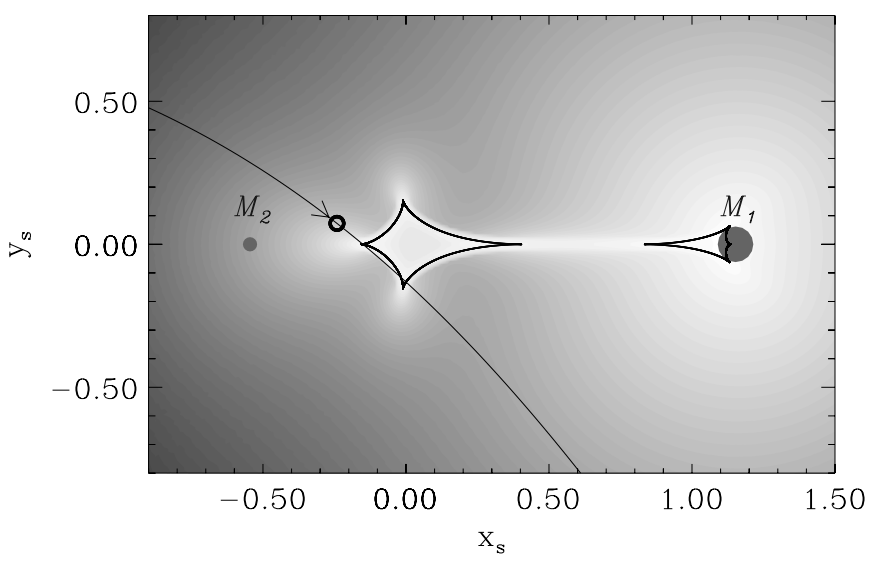

Figure 2. Geometry of the lens system. The closed figures composed of concave curves represent the caustic and the line with an arrow is the source trajectory. $M_{1}$ and $M_{2}$ represent the binary lens components, where $\mathrm{M} 1$ is the heavier one. Grayscale represents the lensing magnification where the brighter tone denotes higher magnification. All lengths are scaled by the Einstein radius corresponding to the total mass of the binary lens.

parallax $\pi_{\mathrm{E}}$ is related to the mass and the distance to the lens by $M_{\text {tot }}=\theta_{\mathrm{E}} /\left(\kappa \pi_{\mathrm{E}}\right)$ and $D_{\mathrm{L}}=\mathrm{AU} /\left(\pi_{\mathrm{E}} \theta_{\mathrm{E}}+\pi_{\mathrm{S}}\right)$, respectively. Here $\kappa=4 G /\left(c^{2} \mathrm{AU}\right), \pi_{\mathrm{S}}=\mathrm{AU} / D_{\mathrm{S}}$ is the parallax of the source star, and $D_{\mathrm{S}}$ is the distance to the source star. The source is in the Galactic bulge and thus its distance is known. Considering the mass distribution of the Galactic bulge and the projected source location, we estimate that $D_{\mathrm{S}}=7.60 \mathrm{kpc}$, corresponding to $\pi_{\mathrm{S}}=0.132$ mas.

For the full characterization of the physical parameters, it is necessary to additionally determine the Einstein radius, which is given by $\theta_{\mathrm{E}}=\theta_{*} / \rho_{*}$. The normalized source radius $\rho_{*}$ is measured by analyzing the caustic-crossing parts of the light curve that are affected by finite-source effects. The angular source radius $\theta_{*}$ is estimated from the source type that is determined based on its de-reddened color and brightness. For this we first calibrate the color and brightness by using the centroid of bulge giant clump as a reference (Yoo et al. 2004), for which the de-reddened brightness $I_{0, \mathrm{c}}=14.45$ at the Galactocentric distance (Nataf et al. 2013) and color 
Table 3

Physical Parameters

\begin{tabular}{lccr}
\hline \hline Parameters & \multicolumn{2}{c}{ Parallax } & Orbit+Parallax \\
\cline { 2 - 3 } & $\left(u_{0}>0\right)$ & $\left(u_{0}<0\right)$ & $0.024 \pm 0.002$ \\
\hline Total mass $\left(M_{\odot}\right)$ & $0.024 \pm 0.002$ & $0.024 \pm 0.002$ & $0.022 \pm 0.002$ \\
Primary mass $\left(M_{\odot}\right)$ & $0.023 \pm 0.002$ & $0.022 \pm 0.002$ & $1.82 \pm 0.16$ \\
Companion mass $\left(M_{\mathrm{J}}\right)$ & $1.89 \pm 0.19$ & $1.85 \pm 0.19$ & $0.86 \pm 0.02$ \\
Projected separation $(\mathrm{AU})$ & $0.89 \pm 0.03$ & $0.87 \pm 0.03$ & $1.73 \pm 0.12$ \\
Distance $(\mathrm{kpc})$ & $1.79 \pm 0.12$ & $1.76 \pm 0.13$ & \\
Height above plane $(\mathrm{pc})$ & $106 \pm 7$ & $106 \pm 7$ & $-59 \pm 8$ \\
Velocity, rotation direction $\left(\mathrm{km} \mathrm{s}^{-1}\right)$ & $-2 \pm 8$ & $-16 \pm 6$ & \\
Velocity, vertical direction $\left(\mathrm{km} \mathrm{s}^{-1}\right)$ & $17 \pm 6$ & & \\
\hline
\end{tabular}

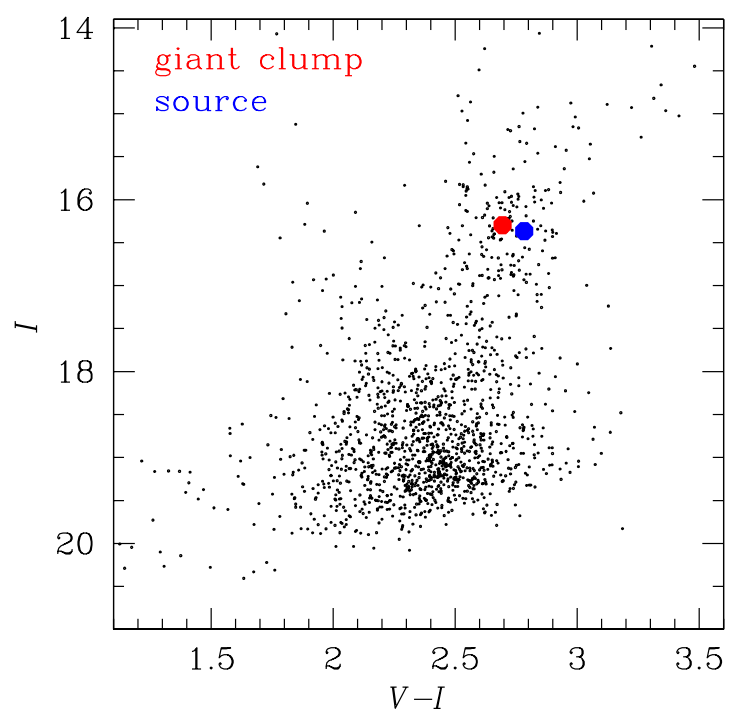

Figure 3. Location of the lensed source star in the color-magnitude diagram (blue dot) relative to the centroid of the red clump (red dot). The magnitude and color are the instrumental scale of the OGLE III data photometry.

(A color version of this figure is available in the online journal.)

$(V-I)_{0, \mathrm{c}}=1.06$ (Bensby et al. 2011) are known. We then translate $V-I$ into $V-K$ color by using the color-color relations (Bessell \& Brett 1988) and then find $\theta_{*}$ using the relation between the $V-K$ and the angular radius (Kervella et al. 2004). Figure 3 shows the location of the source star in the color-magnitude diagram of stars in the same field obtained by the OGLE III experiment. It is found that the source is a $\mathrm{K}$-type giant with an angular radius $\theta_{*}=6.89 \pm 0.60 \mu \mathrm{as}$. The estimated Einstein radius is $\theta_{\mathrm{E}}=0.29 \pm 0.03$ mas. Combined with the measured Einstein timescale $t_{\mathrm{E}}$, the relative lens-source proper motion is $\mu=\theta_{\mathrm{E}} / t_{\mathrm{E}}=4.02 \pm 0.37$ mas per year.

In Table 3, we present the determined physical parameters of the lens. Since the parallax solutions with $u_{0}>0$ and $u_{0}<0$ are degenerate, we present the physical parameters resulting from both solutions. Similarly, we also present the parameters resulting from the orbit+parallax solution. The mass of the companion is twice that of the Jupiter. According to the best-fit parallax model, the mass of the primary is $0.022 \pm 0.002 M_{\odot}$. This is firmly below the hydrogen-burning limit of $0.08 M_{\odot}$ and thus the primary is a BD. The lens is located at a distance $D_{\mathrm{L}}=1.76 \pm 0.13 \mathrm{kpc}$ from the Earth toward the Galactic center. Then the projected separation between the lens components is $\ell_{\perp}=s D_{\mathrm{L}} \theta_{\mathrm{E}}=0.87 \pm 0.03 \mathrm{AU}$.

We also show the height above the Galactic plane $z$ and the transverse velocity $\left(v_{l}, v_{b}\right)$ in the directions of Galactic rotation and Galactic north pole, respectively. To find the latter two, we measure the source proper motion $\left(\mu_{N}, \mu_{E}\right)_{\mathrm{S}}=$ $(-0.20 \pm 0.65,0.02 \pm 0.65)$ mas $\mathrm{yr}^{-1}$ relative to the Galactic bar, and correct for the bar proper-motion gradient (Gould \& Yee 2013). These kinematic variables are the only ones that differ significantly between the two solutions resulting from the ecliptic degeneracy with $u_{0}>0$ and $u_{0}<0$. However, both sets of $\left(v_{l}, v_{b}\right)$ as well as $z$ are consistent with a lens age in the range $1-10 \mathrm{Gyr}$, i.e., much older than BDs of this mass found in imaging studies.

\section{DISCUSSION}

The properties of the OGLE-2012-BLG-0358L system are relatively extreme compared to other binaries with BD hosts. In particular, the separation is a factor $\sim 15$ and $\sim 40$ times smaller than those of 2MASS 1207-3932 and 2MASS 0441-2301, respectively, and the mass ratio of is a factor $\sim 2$ and $\gtrsim 3$ times smaller than the mass ratios of these systems. Systems with such extreme properties may be difficult to form via conventional binary BD formation mechanisms (e.g., Bate 2012), suggesting an alternative scenario where the companion formed in the protoplanetary disk of the host BD. Surveys for disks around young BD have found some systems with inferred disk masses up to and even slightly exceeding $\sim M_{\mathrm{J}}$ (Harvey et al. 2012), although these are relatively rare and the inferred masses are subject to considerable uncertainty. Such massive disks are likely to be near the limit of stability (e.g., Lodato et al. 2005), arguing for a gravitational instability formation scenario rather than core accretion. On the other hand, the relatively close separation may pose a challenge for gravitational instability. Clearly, additional theoretical work is needed to explore the viability of planet formation in BD protoplanetary disks, either by the gravitational instability or core accretion mechanism. For this it is essential to find more binaries with BD hosts in wide ranges of mass ratios and separations.

Microlensing surveys for exoplanets are well-suited to detect planetary companions to very faint, low-mass stars and old BDs, systems which are difficult to discover via other methods. The last two decades have witnessed tremendous progress in microlensing experiments, which have enabled a nearly 10 fold increase in the observational cadence, resulting in an almost 100 fold increase in the event detection rate. With this observational progress, the number of $\mathrm{BD}$ events with precisely measured physical parameters is rapidly increasing (Shin et al 2013; Choi et al. 2013). Furthermore, a new survey based on a network of multiple telescopes equipped with large format cameras is planned to achieve an even higher cadence of more than 100 per day. Hence, starting from the system reported in this work, many additional BD hosts will be surveyed via microlensing. The discovery of additional close separation BD/planet systems 
with even more extreme mass ratio systems from these surveys will provide important empirical constraints on the ubiquity and mechanisms of planet formation around these hosts.

Work by C.H. was supported by the Creative Research Initiative Program (2009-0081561) of the National Research Foundation of Korea. The OGLE project has received funding from the European Research Council under the European Community's Seventh Framework Programme (FP7/2007-2013)/ERC grant agreement No. 246678. The MOA experiment was supported by grants JSPS22403003 and JSPS23340064. T.S. acknowledges support from JSPS 24253004. T.S. is supported by the grant JSPS23340044. Y.M. acknowledges support from the JSPS grants JSPS23540339 and JSPS19340058. A.G. and B.S.G. acknowledge support from NSF AST-1103471. B.S.G., A.G., and R.W.P. acknowledge support from the NASA grant NNX12AB99G. S. Dong's research was performed under contract with the California Institute of Technology funded by NASA through the Sagan Fellowship Program. K.A., D.B., M.D., K.H., M.H., S.I., C.L., R.S., and Y.T. are supported by the NPRP grant NPRP-09-476-1-78 from the Qatar National Research Fund (a member of the Qatar Foundation). M.D. is a Royal Society University Research Fellow. K.H. is a Royal Society Leverhulme Trust Senior Research Fellow. C.S. received funding from the European Union Seventh Framework Programme (FP7/2007-2013) under grant agreement No. 268421. The research leading to these results has received funding from the European Community's Seventh Framework Programme (/FP7/2007-2013/) under grant agreement No. 229517.

\section{REFERENCES}

Albrow, M. D., Beaulieu, J.-P., Caldwell, J. A. R., et al. 2000, ApJ, 534, 894 Alcock, C., Allsman, R. A., Alves, D., et al. 1995, ApJL, 454, L125
Apai, D., Pascucci, I., Bouwman, J., et al. 2005, Sci, 310, 834 Bate, M. R. 2012, MNRAS, 419, 3115

Bennett, D. P., Rhie, S. H., Nikolaev, S., et al. 2010, ApJ, 713, 837

Bensby, T., Adén, D., Meléndez, J., et al. 2011, A\&A, 533, 134

Bessell, M. S., \& Brett, J. M. 1988, PASP, 100, 1134

Bond, I. A., Abe, F., Dodd, R. J., et al. 2001, MNRAS, 327, 868

Boss, A. P. 1997, Sci, 276, 1836

Cameron, A. G. W. 1978, M\&P, 18, 5

Chauvin, G., Lagrange, A.-M., Dumas, C., et al. 2004, A\&A, 425, L29

Choi, J.-Y., Han, C., Udalski, A., et al. 2013, ApJ, 768, 129

Dominik, M. 1998, A\&A, 329, 361

Dong, S., Gould, A., Udalski, A., et al. 2009, ApJ, 695, 970

Durisen, R. H., Boss, A. P., Mayer, L., et al. 2007, in Protostars and Planets V, ed. B. Reipurth, D. Jewitt, \& K. Keil (Tucson, AZ: Univ. Arizona Press), 607

Gould, A. 1992, ApJ, 392, 442

Gould, A. 2004, ApJ, 606, 319

Gould, A., Udalski, A., An, D., et al. 2006, ApJL, 644, L37

Gould, A., \& Yee, J. C. 2013, ApJ, 767, 42

Han, C., \& Gould, A. 1997, ApJ, 480, 196

Harvey, P. M., Henning, T., Liu, Y., et al. 2012, ApJ, 755, 67

Kervella, P., Thévenin, F., Di Folco, E., et al. 2004, A\&A, 426, 297

Kuiper, G. P. 1951, PNAS, 37, 1

Lodato, G., Delgado-Donate, E., \& Clarke, C. J. 2005, MNRAS, 364, L91

Luhman, K. L. 2012, ARA\&A, 50, 65

Luhman, K. L., Adame, L., D’Alessio, P., et al. 2005, ApJL, 635, L93

Luhman, K. L., Adame, L., D’Alessio, P., et al. 2007, ApJ, 666, 1219

Nataf, D. M., Gould, A., Pinsonneault, M. H., et al. 2013, ApJ, 766, 77

Penny, M. T., Mao, S., \& Kerins, E. 2011, MNRAS, 412, 607

Pollack, J. B., Hubickyj, O., Bodenheimer, P., et al. 1996, Icar, 124, 62

Ricci, L., Isella, A., Carpenter, J. M., et al. 2013, ApJL, 764, L27

Ricci, L., Testi, L., Natta, A., et al. 2012, ApJL, 761, L20

Shin, I.-G., Han, C., Gould, A., et al. 2012, ApJ, 760, 116

Shin, I.-G., Sumi, T., Udalski, A., et al. 2013, ApJ, 764, 64

Shin, I.-G., Udalski, A., Han, C., et al. 2011, ApJ, 735, 85

Skowron, J., Udalski, A., Gould, A., et al. 2011, ApJ, 738, 87

Smith, M. C., Mao, S., \& Paczyński, B. 2003, MNRAS, 339, 925

Sumi, T., Abe, F., Bond, I. A., et al. 2003, ApJ, 591, 204

Todorov, K., Luhman, K. L., \& McLeod, K. K. 2010, ApJL, 714, L84

Tsapras, Y., Street, R., Horne, K., et al. 2009, AN, 330, 4

Udalski, A. 2003, AcA, 53, 291

Yoo, J., DePoy, D. L., Gal-Yam, A., et al. 2004, ApJ, 603, 139 